\title{
Challenges and enablers of the embedded researcher model
}

\section{Abstract}

Purpose: The embedded researcher is a healthcare-academic partnership model in which the researcher is engaged as a core member of the healthcare organisation to undertake clinical research and build clinicians' capacity to understand and apply research findings. While this model has potential to support evidence translation, there is a paucity of evidence in relation to the specific challenges and strengths of the model. The aim of this study was to map the barriers and enablers of the model from the perspective of embedded researchers in Australian public hospitals, and compare the responses of embedded researchers primarily managed within the hospital setting versus those managed by a University.

Design: 104 embedded researchers from Australian healthcare organisations completed an online survey. Both purposive and snowball sampling strategies were used to identify current and former embedded researchers. This paper reports on responses to the open-ended questions in relation to barriers and enablers of the role, the available support, and recommendations for change. Thematic analysis was used to describe and interpret the breadth and depth of responses and common themes.

Findings: Key barriers to being an embedded researcher in a public hospital included a lack of research infrastructure and funding in the healthcare organisation, a culture that does not value research, a lack of leadership and support to undertake research, limited access to mentoring and career progression and issues associated with having a dual affiliation. Key enablers included supportive colleagues and executive leaders, personal commitment to research and research collaboration including formal health-academic partnerships.

Research implications: To support the embedded researcher model, broader system changes are required, including greater investment in research infrastructure and healthcare-academic 
partnerships with formal agreements. Significant changes are required, so that healthcare organisations appreciate the value of research and support both clinicians and researchers to engage in research that is important to their local population.

Originality: This is the first study to systematically investigate the enablers and barriers of the embedded researcher model.

Keywords: Embedded research, evidence translation, healthcare-academic partnerships, clinician researchers, research culture 


\section{Introduction}

Although research evidence and evidence based clinical guidelines are being produced at an increasing rate, evidence is not always translated into clinical care (Curtis et al., 2017; Runciman et al., 2012; Miller et al., 2016). As an example, a large nationwide Australian study assessed clinician compliance with evidence based guidelines for 22 conditions and found that patients only received 'appropriate care' $57 \%$ of the time (Runciman et al., 2012). Only around half of research evidence is ever translated into practice, and of the evidence that is translated it has been reported that, in some contexts, it can take up to 17 years for the new practice to be adopted routinely (Bauer et al., 2015).

The implementation of new practices into routine care is a complex process that takes considerable effort (Barnes et al., 2015; Kitson et al., 2017; Olswang and Prelock, 2015). Evidence translation is associated with a wide range of individual and organisational factors and processes (e.g. social, behavioural, economic, management) that may hinder or support implementation (Greenhalgh, 2018; Curtis et al., 2017). Given the unique context of different healthcare organisations, for evidence to be adopted, interventions need to be adapted to the local context and address local priorities and issues (Braithwaite et al., 2018; Kitson et al., 2017). Merely disseminating knowledge through journals and clinical guidelines, which relies on the clinician to read, interpret and apply the findings, is not enough to ensure evidence adoption (Olswang and Prelock, 2015; Greenhalgh, 2018).

To be useful, evidence needs to actively translated into practice (Holmes et al., 2017; Braithwaite et al., 2018; loannidis, 2016; Barnes et al., 2015). To support this process, over the last 15 years, implementation scientists have investigated ways to support evidence implementation, and developed numerous theories, frameworks and models (Schaffer et al., 2013; Kitson, 2010; Nilsen, 2015; Damschroder et al., 2009; Fixsen et al., 2005). It is increasingly recognised within this body of work that for evidence to be implemented and sustained, researchers and healthcare providers must 
work together (Kitson et al., 2013; Holmes et al., 2017; Ellen et al., 2013). However, as in Australia and elsewhere, health research and health care provision are generally viewed as independent and the responsibility of separate agencies and governments (Barnes et al., 2015; Rycroft-Malone et al., 2013). Consequently, collaboration between researchers and healthcare providers is seldom prioritised. Those generating evidence (i.e. the researchers) continue to work in isolation from those implementing research findings (i.e. clinicians and health service managers) (Powell et al., 2013; Kitson et al., 2017; Stetler et al., 2011).

To help bridge the gap between research and practice, one emerging partnership model that is gaining attention is the 'embedded researcher' model (Marshall, 2014a; Bannister and Hardill, 2013; Cheetham et al., 2018; Vindrola-Padros et al., 2017). The 'embedded researcher model' refers to a model of collaboration where the researcher is employed as a core member of the health service delivery team (Marshall et al., 2014; Lewis and Russell, 2011; McGinity and Salokangas, 2014). Embedded researchers can be conceptualised as knowledge brokers (Bornbaum et al., 2015; LaRocca et al., 2012) that collaborate with clinical teams to undertake research, and build clinicians' capacity to understand and apply research findings (McGinity and Salokangas, 2014; Vindrola-Padros et al., 2017).

The premise of the embedded research model is that knowledge that is collected and created 'on the ground' will provide better insight into the issues affecting practitioners, managers and service users, be more relevant to the local context and be more easily incorporated into practice changes (Ward et al., 2009; Greenhalgh and Wieringa, 2011; Bannister and Hardill, 2013). By being immersed in the healthcare organisation, the embedded researcher has insider knowledge and access to contextual information not readily available to outsiders (Marshall et al., 2014; Lewis and Russell, 2011; Walsh, 2011), and can gain greater understanding of the pressures faced by the organisation and tailor improvement strategies accordingly (McGinity and Salokangas, 2014; Marshall et al., 
2014). Due to their immersion within the organisation, embedded researchers can produce research that is more relevant to the 'end user' (Lewis and Russell, 2011) and are well placed to build the organisation's research capacity by promoting a reflexive learning culture, establishing a research culture and teaching evaluation and research skills (Marshall et al., 2014; Wenke et al., 2017).

The adoption of embedded researchers varies considerably between organisations. While some conceptualise embedded researchers as having a dual healthcare and academic affiliation (VindrolaPadros et al., 2019), this is not always the case and some embedded researchers are entirely employed by a healthcare organisations (Vindrola-Padros et al., 2017; McGinity and Salokangas, 2014). Within the Australian context, some embedded researchers are funded by Universities, some are funded by the healthcare organisation through core funding or grants, and others are funded as a partnership between a healthcare and academic institutions (Coates and Mickan, 2019). The aim and purpose of the embedded researcher role can vary considerably but generally includes knowledge production, knowledge translation and research capacity building (McGinity and Salokangas, 2014; Vindrola-Padros et al., 2017; Coates and Mickan, 2019).

While the embedded researcher model may enhance evidence uptake by bridging the gap between healthcare research and health service delivery, the potential risks and challenges of the model are increasingly recognised (Vindrola-Padros et al., 2017; Lewis and Russell, 2011; Hackett and Rhoten, 2011; Churruca et al., 2019). Researchers, clinicians, and health service managers have different agendas, ways of working, priorities and timeframes, which can be in conflict (Marshall et al., 2016; Vindrola-Padros et al., 2017). Examples of potential challenges include embedded researchers feeling bound by internal regulations that prevent them from publishing negative findings (Hackett and Rhoten, 2011), and they may feel conflicted between their commitments to the healthcare organisation and to the academic standards for conducting research (Lewis and Russell, 2011). 
Despite increasing interest in the model, the strengths and challenges for embedded researchers are not well understood (Marshall, 2014b; Vindrola-Padros et al., 2017; Churruca et al., 2019; Barry et al., 2018). To support the development and implementation of this model, a better understanding of risks and strengths as experienced by embedded researchers is needed (Marshall, 2014b; VindrolaPadros et al., 2017; Churruca et al., 2019; Barry et al., 2018). Furthermore, questions are increasingly being raised around the degree of embeddedness, and whether the strengths and challenges vary depending of the degree of 'embeddedness' (Churruca et al., 2019; Barry et al., 2018). More specifically, it is unclear how the challenges and strengths of the model differ from the perspective of embedded researchers who are fully embedded and employed by the healthcare organisations, versus those who have a dual affiliation with a healthcare and academic institution (Churruca et al., 2019; Barry et al., 2018). Within the Australian context, some embedded researchers are primarily affiliated with a healthcare organisation and others with an academic organisation. By necessity, individuals need to belong to one institution and adhere to its human resource and work practices, despite often having joint contracts and being expected to work across two organisations.

The aim of this study was to map the challenges and enablers of the model from the perspective of embedded researchers in Australian public hospital settings, and compare the barriers and enablers reported by those with a primary academic versus healthcare affiliation. We defined an embedded researcher as someone with research qualifications who is employed for at least $30 \%$ of their time (0.3FTE) in a healthcare organisation and has research and research capacity building as part of their role, with or without a dual affiliation with an academic institution. By comparing the responses of embedded researchers with a primary academic versus healthcare affiliation we sought to provide insight into whether being primarily or entirely embedded in a healthcare organisation was associated with more or different strengths and challenges. 
The findings presented here are part of a larger exploratory survey study into the role and experiences of embedded researchers. As outlined elsewhere (Mickan and Coates, under review), the embedded researchers that participated in this study conducted personal implementation research (i.e. implement and evaluate interventions or service models in a public hospital) and engaged in capacity building. The capacity building component of their role involved developing skills and knowledge in clinicians to understand and apply research findings and participate in research (e.g. through mentoring and teaching) (Mickan and Coates, under review). The findings reported here are limited to the qualitative results concerned with the strengths and challenges of the role, the available support and embedded researchers' recommendations for improvement.

\section{Method}

An online anonymous survey was developed for researchers embedded in Australian public hospitals to identify key aspects of their role and to capture their perceptions of current enablers and barriers of their role. An online survey method was used to reach a larger population of embedded researchers than interviews would have allowed, and to collect a broad range of both qualitative and quantitative data. The aim of the qualitative component of this study was to describe the enablers and barriers of embedded research, across a diverse group of embedded researchers affiliated with a range of different public healthcare organisations and universities. As this is an under-researched area, we wanted to gain insight into their experiences as a larger group.

A range of questions were developed and piloted with four embedded researchers known to the authors, using multiple choice and Likert scales for quantitative questions and open-ended questions to develop new insights into the experiences of embedded researchers. The design of the survey was informed by a purposive (and unpublished) review of the embedded research literature, the authors' experience as embedded researchers and feedback received during the pilot process. Face validity of the questionnaire was established by seeking feedback from five embedded researchers 
who reviewed the questions and confirmed their appropriateness (Bolarinwa, 2015). The survey was implemented using REDCap, a customizable informatics systems-based web software (REDCap, 2020).

A purposive sample of current and former embedded researchers were invited to participate via email, which contained information about the aim and purpose of the study and an online link to the survey (Babbie, 2015). Both authors personally invited embedded researchers known to them from their relevant local and national networks to participate (69 invitations sent). Using a snowball strategy, these individuals were asked to pass on the survey to other embedded researchers in their networks (Bryman, 2016). While embedded researchers were able to self-identify, the email invitation clearly stipulated that only those employed by a healthcare organisation at a minimum of $30 \%$ of their time $(0.3 \mathrm{FTE})$ with research capacity building as part of their role met criteria. No minimum duration of employment was stipulated and no incentive for participation was provided. Data was collected over a two months period, from the $23^{\text {rd }}$ of January until the $23^{\text {rd }}$ of March 2019.

This paper reports on participant responses to the open-ended questions in relation to the enablers and challenges of the role, and the available support; specifically:

- What are the top challenges/barriers to achieving the goals/purpose of your role?

- What are the top enablers helping you to achieve your purpose?

- How does your line manager within the healthcare organisation support your work?

- Where do you gain your personal mentoring, support?

- What could be changed to improve the culture of the healthcare organisation to support you to achieve your purpose?

In addition, we briefly report on respondents' characteristics (such as primary affiliation, discipline, years of experience and types of roles) to provide context. To encourage frank disclosure in relation 
to the challenges of the role, respondents were not asked to disclose their name, nor the name of their organisation.

Data was exported from REDCap into Excel 2016, and qualitative responses were copied into Word 2016 for analysis. Following the steps outlined by Braun and Clarke (40), we used thematic analysis to describe the range of perceived barriers and facilitators, and to interpret meaningful themes. Specifically, qualitative responses were coded to identify initial themes, which were then reviewed across all respondents to generate enduring themes. Themes were identified and named to convey the explicit (or surface) meaning of what each respondent wrote (Braun and Clarke, 2006). The number of times all respondents referred to these themes was also recorded as a frequency of mention. Given that there was a consistent set of open-ended questions, there is an assumption that themes which were reported more frequently may be more important than those mentioned less frequently. The frequency in which each theme was reported by embedded researchers with a primary healthcare affiliation was compared to those with a primary academic affiliation.

To ensure rigour, the data was analysed in two rounds. In the first instance, both authors analysed the data independently and came together to discuss the themes and discrepancies. From this a coding frame was developed and a second round of analysis was conducted by one author (DC) using this framework. Further modification of the framework based on the second round of analysis was discussed and agreed upon by both authors. Ethics approval for this study was received by University of Technology Sydney human research ethics committee (HREC reference number ETH182901).

\section{Results}


A total of 104 embedded researchers, who worked in Australian public hospitals completed the online survey. Public hospitals provide care without cost to Australian citizens and permanent residents, based on their clinical needs. Most respondents identified their professional background as nursing and midwifery ( $n=37)$, allied health $(n=36)$ and medicine $(n=27)$. A further 4 identified as from a non-traditional health discipline such as social science. An equal number of embedded researchers reported a primary academic $(n=52)$ versus a primary healthcare affiliation $(n=52)$. Of the 52 embedded researchers who were primarily employed by a healthcare organisation, most $(n=35,68 \%)$ were fully funded by their healthcare organisation. Of the 52 embedded researchers with a primary academic affiliation, only $4.7 \%$ were fully funded by a healthcare organisation, with the majority paid for/reimbursed by a healthcare organisation at between $30-50 \%$ ( $n=42,81.4 \%$ ). While some had been in their role for more than 16 years, almost half reported less than 2 years' experience as an embedded researcher. Most (68\%) reported belonging to a clinical department and a smaller proportion were aligned to a research or quality improvement unit. Further detail on the role of embedded researchers is outlined elsewhere (Mickan and Coates, under review).

\section{What are the barriers of embedded research?}

Analysis of responses to the question "What are the top challenges/barriers to achieving the goals/purpose of your role?" identified 11 key themes or barriers experienced by embedded researchers, specifically research not being sufficiently valued in healthcare organisations, lack of support from line managers, insufficient time/competing clinical demands, inadequate research resources and funding in the healthcare organisation, lack of research infrastructure in the healthcare organisation, complex research governance processes, limited research skills of clinicians, limited access to academic mentoring, limited opportunity for career progression, little recognition of implementation science/health service research, and challenges associated with having a dual affiliation (see Table 1). While most challenges were raised by both embedded researchers with a primary healthcare and those with a primary academic affiliation, the challenges 'lack of 
management support' and 'limited career progression' appeared specific to those with a primary healthcare affiliation, and the challenges associated with a dual affiliation appeared unique to those with a primary academic affiliation. A short description of each of the 11 barriers is described and illustrated by direct quotations in the text and in Table 1, and the frequency of mention of the themes is illustrated in Figure 1.

\section{Insert Figure 1: Barriers of embedded research}

\section{Research not sufficiently valued in healthcare organisation}

The research culture, or lack thereof, of the healthcare organisation was highlighted as a key barrier by the majority of embedded researchers, in particular those with a primary healthcare affiliation (57 mentions, 42 with primary healthcare affiliation vs 15 with academic affiliation). Respondents commented that research is not sufficiently valued as a means to inform clinical practice. Comments such as "My research expertise is not valued", "There is a cultural resistance to research and evidence", "Research is viewed as interfering with clinical work" and "The healthcare service sees research as the responsibility of the university (including all funding for it)" were common.

Both frontline clinicians and executive leadership were described as "not engaged in research". Clinicians were described as "resistant to research", "hard to engage", "lacking in motivation" and "not interested in research". Respondents commented that engaging clinicians, in particular medical staff, in research projects was very difficult. The executive leadership was described as "unsupportive" and lacking in research knowledge and experience. Respondents commented that the capacity of clinicians to drive and participate in research was undervalued by the organisation, and that there was an expectation for research to be undertaken for clinical staff rather than with clinical staff. 


\section{Lack of line management support}

A lack of support from line managers was another key barrier, reported by embedded researchers with a primary healthcare affiliation (45 mentions), the majority of whom were embedded in a clinical department and reported to a clinical manager. They described barriers to research, as " $m y$ manager's lack of understanding of the research process" and "my manager's lack of interest in my work". Respondents explained that their managers did not understand research or the skills required, and that they had unrealistic expectations of timeframes. Some respondents described their managers as hindering research by being overly "risk averse" and "lacking in imagination". Some noted that their manager was not interested in their work and that they received little to no support. As commented by one respondent:

"I have little contact with my line manager within the health care organisation and they show little interest or knowledge of my work".

\section{Insufficient time/competing clinical priorities}

Competing clinical priorities were described by many embedded researchers to reduce time for research, and this was a greater challenge for those with a primary healthcare affiliation ( 35 versus 19 mentions). Respondents reported that they were juggling too many competing demands, with comments such as "there is a lack of time for research as well as clinical and management priorities" and "insufficient time to balance research with competing clinical demands" common. Respondents explained that there was inadequate protected time for clinical research, and that there was often no funding to release clinicians to undertake research. Some respondents noted that they end up doing much of their research in their own time (i.e. not paid). 


\section{Inadequate research resources and funding in the healthcare organisation}

Inadequate research resources were an issue identified equally by those with a primary healthcare and academic affiliation ( 25 vs 24 mentions). A lack of research positions as well as funding for open access publications or conference attendance were key challenges. Embedded researchers with primary healthcare affiliation highlighted the challenges associated with not having secure research positions and limited ongoing research employment opportunities as many were on temporary contracts often funded by grants. Embedded researchers with primary academic affiliation highlighted the challenges associated with not having access to research assistants or research support staff within the healthcare organisation, and that they spent too much time chasing funding to conduct healthcare research.

\section{Lack of research infrastructure in the healthcare organisation}

Respondents, in particular those with a primary academic affiliation, highlighted the lack of research infrastructure as a key barrier (13 vs 6 mentions). The specific challenges included limited access to technology that supports research such as research software, insufficient electronic storage within Health organisations for research data, firewalls on healthcare organisation computers, and blocked access to external research websites as well as difficulties in establishing and recruiting to research positions (for example, research awards not available through the healthcare organisation). Limitations in relation to the physical environment were also highlighted, with some respondents commenting that the office space was not ideal, and that there was not enough space. Those with a dual affiliation commented that having two offices was a challenge.

\section{Complex research governance processes}

The time and processes for research governance, i.e. the system of administration and supervision through which research is managed and accountability is assured, were highlighted as key barriers, in particular by respondents with a primary academic affiliation (12 vs 4 mentions). Comments such 
as ", "approval for ethics and Governance is overly onerous", and "disproportionate ethics and governance for low-risk research" were common.

\section{Limited research skills of clinicians}

Limited research skills of clinicians was another key challenge, identified more frequently by those with a primary academic affiliation (13 vs 6 mentions). Comments such as "Clinicians lack knowledge of research" and "clinicians aren't trained in research so therefore don't engage" were common.

\section{Limited access to academic mentoring}

Limited access to academic mentoring was a barrier mostly identified by respondents with a primary healthcare affiliation (12 vs 4 mentions). Respondents commented that there was a lack of "access to mentoring" with little access to "role models" or "supervision". Comments such as "There isn't really any opportunity for academic support or mentoring within my organisation" were common. One respondent commented that she did not have access to mentoring because "I have a clinical manager as my boss who doesn't have research skills so can't mentor me".

\section{Limited opportunity for career progression}

Respondents with a primary healthcare affiliation also identified limited opportunity for career progression as a barrier to embedded research (10 mentions). These respondents commented that they did not have a research career pathway within the healthcare organisation, and that there are very few employment opportunities that combine research and clinical practice. They also noted that researchers primarily affiliated with a healthcare organisation experience a lack of pay equivalent to academic positions.

\section{Little recognition of implementation science/health service research}


Another barrier identified by respondents with a primary academic and healthcare affiliation ( 2 vs 6$)$ was the belief that health service research and implementation science was undervalued.

Respondents commented that health service research was undervalued by their university as well as funding bodies. As commented by one respondent with a primary healthcare affiliation:

"There is a lack of funding for and recognition of knowledge translation as a legitimate research activity. I am constantly told that my work is too researchy by those in quality improvement, and not researchy enough by those in research!"

\section{Challenges associated with having a dual affiliation}

Respondents with a primary academic affiliation commented that there is a tension between the expectations and needs of the healthcare organisation and the academic institution (22 mentions). Comments such as "the two organisations have different goals and expectations" were common. More specifically, respondents explained that health services research is often hospital specific and does not meet the universities' requirement of high impact. As noted by one respondent:

"Health service research is usually low level and institution specific which does not align with university expectations of wide scale impact and high importance".

Respondents also noted that the partnership model between the university and the hospital was not well developed and there was a need for "better integration between research, clinical service and education". They noted that embedded researchers fall between the gap of academia and healthcare and are not supported by either organisation. As commented by one respondent: 
"The university employs me but provides essentially no support and never asks me about my role. The health organisation focusses on staff specialists as they believe the universities are looking after the clinical academic. So we operate in a vacuum".

Insert Table 1: Barriers and enablers to embedded research as reported by respondents

\section{What are the enablers of embedded research?}

Analysis of the responses to the question "What are the top enablers helping you to achieve your purpose?" identified eight key enablers, some of which are the converse of the barriers outlined above. Specifically, enablers identified were: access to research colleagues and mentors, supportive executive leadership, personal commitment to research, research collaborations and healthcareacademic partnerships, supportive clinical managers, enthusiasm and research engagement from clinicians, access to research funds, and access to research supports (e.g. research office in the healthcare organisation) (Figure 2, Table 1). Overall enablers were less frequently mentioned than barriers (i.e. the question regarding barriers generated a total of 315 barriers across all respondents, and the question about enablers generated 186 enablers). A short description of each of the enablers is described and illustrated by direct quotations in the text and in Table 1.

Insert Figure 2: Enablers of embedded research

\section{Supportive executive leaders within the healthcare organisation}

Research leadership within the healthcare organisation was the most frequently identified enabler of embedded research, by both those with a primary healthcare and academic affiliation (18 and 11 
mentions). Specifically, respondents identified "supportive executive leaders", "supportive directors" and other "lead people who are interested to see a research focus within the service" as enablers.

\section{Research colleagues and mentors}

The support received from research colleagues and mentors was the most frequently identified enabler of embedded researchers, specifically by those with a primary healthcare affiliation ( 21 and 13 mentions). Respondents identified their research colleagues within the healthcare organisation, affiliated universities or other research networks as key sources of support. Those that had access to mentoring, highlighted this as critical.

\section{Personal commitment to research}

The third most frequently mentioned enabler was the respondents' personal commitment to research, in particular for those with a primary healthcare affiliation (19 vs 7 mentions). Respondents commented that they achieve their goals as embedded researchers because of their own "commitment to research", "passion and enthusiasm to improve health services", "own drive and self-belief", "self-motivation", "personal resilience" and "tenacity". Comments such as "I achieve my goals because I love research and I'm resilient" were common.

\section{Research collaborations and healthcare-academic partnerships}

Research collaborations, in particular healthcare-academic partnerships, were also highlighted as key enablers of embedded research, by both those with a primary healthcare and academic affiliation (15 vs 11 mentions). Respondents identified the commitment to a healthcare-academic partnership at the hospital executive as well as the university as enabling embedded research (e.g. formal agreements in relation to the dual relationship of embedded researchers). More specifically, respondents with a primary healthcare affiliation highlighted the importance of the support received by the university, such as access to university resources (e.g. library databases) and opportunities for 
networking. Respondents with a primary academic affiliation highlighted the value of being embedded in the healthcare organisation, in particular as it facilitates ready access to clinicians and patients to participate in research.

\section{Supportive clinical managers}

Supportive clinical managers was another key enabler, for both those with a primary healthcare and academic affiliation (16 vs 9 mentions). Respondents with a primary healthcare affiliation highlighted the value of "a supportive line manager", noting that being recognised and supported by their manager makes them able to achieve their role. Those with a primary academic affiliation (and academic reporting line), highlighted the importance of clinical managers that are interested and supportive of research. In particular, clinical managers that were research trained and understood the limitations of research were highly valued.

\section{Clinician enthusiasm for research}

Clinician enthusiasm for research was another key enabler, more frequently identified by those with a primary academic affiliation (14 vs 6 mentions). Specifically, respondents highlighted the value of working with clinicians who understand research, were engaged in the process, and were enthusiastic about research. Comments such as "Some clinicians are very interested and supportive of research and that's very motivating" were common.

\section{Financial support/access to resources}

While not mentioned frequently, respondents, particularly those with primary academic affiliation (14 vs 5 mentions) who have managed to access research funds through the healthcare organisation (i.e. hospital research grants, clinical backfill for research) commented that it supported them in their role as embedded researchers. 


\section{Healthcare research facilities and supports}

While mentioned infrequently, healthcare research facilities and supports, such as the hospital library and the research office were also identified as enablers, in particular by respondents with a primary healthcare affiliation (6 vs 1 mentions).

\section{How are managers supporting embedded researchers?}

While many embedded researchers did not feel supported by the organisation/their manager to undertake research, analysis of responses to the question "How does your line manager within the healthcare organisation support your work?" identified key ways healthcare managers support embedded researchers (see Figure 3). Responses to this question were consistent for those with a primary academic versus healthcare affiliation, and are reported together.

Insert Figure 3: How do healthcare managers support embedded research?

The most frequently mentioned way in which healthcare managers supported embedded researchers was by being supportive of healthcare research more broadly (28 mentions). Support was demonstrated by "keeping research on the agenda", "getting funding for research roles", "advocating for research at higher levels", "supporting and facilitating research collaborations" and by "being a champion for research". Respondents also appreciated being given appropriate independence and autonomy (20 mentions), for instance "by giving me freedom to make my own decisions and manage my own workload" and "by giving me a "long leash".

Further ways in which healthcare managers supported embedded researchers included by facilitating the logistics required to support research (e.g. manage human resources related issues, staffing, get office space); by supporting academic activities such as grant application, publications and conference attendance; by allocating appropriate time for research; through regular 
communication and making time for meetings; by valuing the input of the embedded researcher in relation to service improvement; by helping embedded researchers navigate the health system and by celebrating the embedded researchers' successes.

\section{Where do embedded researchers gain mentoring and support?}

The most frequently identified sources of support and mentoring were academic colleagues through university or other academic collaborations ( 56 mentions). This was followed by personal networks such as previous colleagues, friends and family (19 mentions), and fellow clinician researchers (16 mentions). Least frequently, few embedded researchers identified their direct line manager (10 mentions) as a key source of support and mentoring.

Insert Figure 4: Where do embedded researchers gain support and mentoring?

\section{What changes can be made to better support embedded researchers?}

Many respondents commented that significant strategic and cultural change is required to embed research as integral to evidence-based care throughout all levels of the healthcare organisation. Most commonly, they reported that "research needs to become part of normal care, not as something extra". Respondents provided some more tangible suggestions of how the above could be achieved, including embedding research into job descriptions, employing research skilled staff in leadership roles, developing a research strategy for each department, and investing in research infrastructure. These and more recommendations are outlined in Summary box 1.

Insert Summary box 1: Recommendations to better support embedded research 


\section{Discussion}

While the embedded researcher model holds promise in terms of assisting the translation of evidence into practice, consistent with previous studies, our findings indicate that this model comes with a number of challenges (Churruca et al., 2019; Barry et al., 2018; Hackett and Rhoten, 2011; Sim et al., 2018), perhaps in particular for those fully embedded in the health system. While supporting the development of healthcare-academic partnerships, our findings identified significant challenges for healthcare organisations to appreciate the value of research in driving quality care and to support innovative embedded researcher positions with appropriate research-aware leadership and strategic investment in research infrastructure.

This study demonstrated how difficult it can be for embedded researchers to align goals and expectations between organisations with very different cultures and expectations about research. Respondents described a culture in healthcare organisations where research is not valued, executives and managers are not capable/interested/supportive of research, and the practical infrastructure provides inadequate research resources, funding and complex research governance procedures. Further, embedded researchers have variable access to academic mentoring, limited career opportunities and they need to engage with clinicians who have limited research skills and interest. Consequently, competing clinical priorities limit time and funding for research. At the same time, academic organisations opted to progress independently towards high quality research, which they did for, rather than with, clinical staff.

Respondents recognised the need for multifaceted initiatives within both healthcare and academic organisations to support healthcare-academic partnerships to design and translate clinical important research. Many of their comments are well supported by the literature. Respondents described the need to actively address barriers of busy, uninterested clinicians and managers, and engage clinicians' enthusiasm for research. The importance of embedded researchers building clinicians' 
research capacity is well established in improving research cultures within healthcare organisations (Perry et al., 2008; Wenke and Mickan, 2016).

Respondents described supportive executive leaders and clinical managers as key enablers. It is recognised that healthcare managers can positively influence their organisations to support and value research (Perry et al., 2008; Wenke and Mickan, 2016; Janssen et al., 2013), particularly when they experience the benefits. The benefits of doing clinical research include experiential learning, which can improve clinical practice and patient outcomes (Higgins et al., 2010). A recent longitudinal study concluded that first-level leaders play a critical role in implementing evidence-based practice, through creating a workplace climate that expects, supports and rewards clinicians to use evidencebased practices (Williams et al., 2020). Further, recommendations from systematic reviews link research engagement to individual job descriptions and professional development and team level service improvement and practice development activities. Motivational and attitudinal benefits have been described when a critical mass of clinicians engage with research. Overall the literature summarises that research capacity building strategies should be developed and tailored for individuals, teams and organisations (Perry et al., 2008; Wenke and Mickan, 2016; Janssen et al., 2013; Matus et al., 2018). For research to be recognised as core business, it needs to be valued by individual clinicians and managers and embedded within policy.

Our findings highlight a need for healthcare-academic partnership models to be developed to support collaboration between clinicians and researchers that move beyond merely having a dual affiliation. While having a dual affiliation can be protective, it can also be a career development risk for academics, due to the time spent in the field, away from producing traditional academic outputs (Vindrola-Padros et al., 2017; Lewis and Russell, 2011; Vindrola-Padros et al., 2019). Nonetheless, embedded researchers without a dual affiliation report feeling disconnected from their academic peers and not having adequate access to support and mentoring (Vindrola-Padros et al., 2017). 
Recognising the value of dual affiliations, consistent with recommendations put forward by VindrolaPadros (2017) four embedded researchers in this study suggested that the risks associated with a dual affiliation can potentially be managed through clear and formal agreements between the two organisations. Even so, while important, formal agreements alone are not sufficient to develop genuine collaboration between academics and clinicians.

To support the development of effective healthcare-academic partnerships, the literature outlines of range of strategies, such as engaging clinicians in study design, developing shared goals, being flexible and communicating regularly and strategically (Drahota et al., 2016; Pellecchia et al., 2018; Holmes et al., 2017; Barnes et al., 2015). While, like dual affiliation and formal agreements, such strategies are useful, we believe that without changes to the research culture of the healthcare organisation they are hard to implement. Significant changes are required, so that healthcare organisations appreciate the value of research and support both clinicians and researchers to engage in research that is important to their local population. It is important that universities and healthcare organisations develop strategic and operational initiatives to support collaboration between researchers and clinicians, in order to maintain high quality evidence-informed clinical practice (Tran et al., 2017; Marshall et al., 2016; Marshall et al., 2014).

For healthcare-academic partnership to be successful, it is critical that both healthcare organisations and universities invest in embedded researchers and create appropriate research funding and infrastructure to develop and support collaborative research. As outlined in Box 1, the study respondents made a range of recommendations to improve the research culture, capacity and clinical practice of healthcare organisations, such as embedding research into job descriptions of all staff, employing individuals with research skills and qualifications in leadership roles, developing a research strategy for each department, and investing in research resources and infrastructure. Similar recommendations have been made by other studies that highlight the need for greater 
investment in research infrastructure and a research skilled workforce to improve the research culture of healthcare organisations (Barnes et al., 2015; Holmes et al., 2017). While we support a recommendation for greater investment in research resources and infrastructure, given limited funding, the need for support and mentoring expressed by embedded researchers could potentially be responded to by developing and supporting 'embedded researcher' peer support networks (at a state or national level).

The continuing separation between research and health care delivery impedes the effective translation of evidence into practice, and denies the benefits that health organisations could derive from closer engagement in research (Barnes et al., 2015; Hanney et al., 2013). While both organisations are working under increasing resource constraints to achieve their uniquely independent KPIs, there seems to be a limited oversight of the potential benefits to both organisations of the benefits to patients and the community of research informed clinical practice. An important first step for healthcare leaders and policy makers is to make a commitment to evidence based care and implementation research, ensuring appropriate resourcing (Barnes et al., 2015; Holmes et al., 2017). Following on, it will be important to clarify the real benefits in language and KPIs that both organisations value.

\section{Limitations}

This study has a number of limitations. Firstly, given little is known about the embedded researcher model in Australian healthcare, it is unclear how representative our sample is so our findings cannot be generalised. Furthermore, given our snowball sampling approach, we do not know how many embedded researchers were invited to participate, so it is unclear what percentage of the potential respondents contacted participated. This study is also limited to a surface level description of the key challenges and enablers. Given little is known about the experiences of embedded researchers, we opted to seek surface level feedback from a larger group of embedded researcher using surveys 
(i.e. larger than interviews would have allowed), at the expense of a deeper and more complex understanding of the issues using interviews would have allowed. Further qualitative research is required using interviews or focus groups to gain deeper insight into the contextual factors and underlying mechanisms that support or hinder embedded research.

\section{Conclusion}

To the best of our knowledge this is the first study to systematically investigate the enablers and challenges of the embedded researcher model as experienced by embedded researchers. In addition to identifying enablers and barriers of the model more broadly, our findings also contribute to the discussion around the degree of embeddedness, and suggest that both being fully immersed in a healthcare organisation and having a dual affiliation comes with unique challenges. While embedded researcher models where the researcher is fully immersed in healthcare may be desirable (as by being fully immersed provides access to contextual information not readily available to outsiders), this model provides limited access to research mentoring opportunities and career progression. To support the embedded researcher model, broader system changes within the healthcare organisation as well as academia are required. It is hoped that our findings will support or inform the ongoing development and implementation of embedded research and help mitigate potential risks, and enhance collaboration between academia and healthcare organisations. 


\section{References}

Babbie ER. (2015) The practice of social research: Nelson Education.

Bannister J and Hardill I. (2013) Knowledge mobilisation and the social sciences: dancing with new partners in an age of austerity. Contemporary Social Science 8: 167-175.

Barnes R, Holmes B, Lindstrom R, et al. (2015) Evidence-informed healthcare through integration of health research. Healthcare Management Forum 28: 75-78.

Barry D, Kimble L, Nambiar B, et al. (2018) A framework for learning about improvement: embedded implementation and evaluation design to optimize learning. Int J Qual Health Care 30: 10-14.

Bauer MD, L, , Hagedorn H, Smith J, et al. (2015) An introduction to implementation science for the non-specialist. . BMC Psychology 3: 32.

Bolarinwa O. (2015) Principles and methods of validity and reliability testing of questionnaires used in social and health science researches. 22: 195-201.

Bornbaum CC, Kornas K, Peirson L, et al. (2015) Exploring the function and effectiveness of knowledge brokers as facilitators of knowledge translation in health-related settings: a systematic review and thematic analysis. Implementation Science 10: 162.

Braithwaite J, Churruca K, Long J, et al. (2018) When complexity science meets implementation science: a theoretical and empirical analysis of systems change. BMC Medicine 16.

Braun V and Clarke A. (2006) Using thematic analysis in psychology. Qualitative Research in Psychology 3: 77-101.

Bryman A. (2016) Social research methods: Oxford university press.

Cheetham M, Wiseman A, Khazaeli B, et al. (2018) Embedded research: a promising way to create evidence-informed impact in public health? Journal of Public Health 40: i64-i70.

Churruca K, Ludlow K, Taylor N, et al. (2019) The time has come: Embedded implementation research for health care improvement. Journal of Evaluation in Clinical Practice 25: 373-380.

Coates D and Mickan S. (2019) The embedded researcher model in Australian healthcare settings: Comparison by degree of 'embeddedness'. Translational Research.

Curtis K, Fry M, Shaban RZ, et al. (2017) Translating research findings to clinical nursing practice. J Clin Nurs 26: 862-872.

Damschroder L, Aron D, Keith R, et al. (2009) Fostering implementation of health services research findings into practice: a consolidated framework for advancing implementation science. Implementation Science 4: 1-15.

Drahota A, Meza R, Brikho B, et al. (2016) Community-Academic Partnerships: A Systematic Review of the State of the Literature and Recommendations for Future Research. The medibank quarterly 94: 163-214.

Ellen ME, Léon G, Bouchard G, et al. (2013) What supports do health system organizations have in place to facilitate evidence-informed decision-making? a qualitative study. Implementation Science 8: 84.

Fixsen DL, Naoom SF, Blase KA, et al. (2005) Implementation Research: A Synthesis of the Literature, Tampa, FL: University of South Florida, Louis de la Parte Florida Mental Health Institute.

Greenhalgh T. (2018) How to Implement Evidence-Based Healthcare, Oxford, UK: Wiley Blackwell.

Greenhalgh T and Wieringa S. (2011) Is it time to drop the 'knowledge translation' metaphor? A critical literature review. JRSM 104.

Hackett EJ and Rhoten DR. (2011) Engaged, embedded, enjoined: science and technology studies in the National Science Foundation. Sci Eng Ethics 17: 823-838.

Hanney S, Boaz A, Jones T, et al. (2013) Engagement in research: an innovative three-stage review of the benefits for healthcare performance. Health Serv Deliv Res. 1.

Higgins I, Parker V, Keatinge D, et al. (2010) Doing clinical research: The challenges and benefits. Contemp Nurse 35: 171-181.

Holmes B, Best A, Davies H, et al. (2017) Mobilising knowledge in complex health systems: a call to action. Evidence and Policy 13: 539-560. 
loannidis JPA. (2016) Why Most Clinical Research Is Not Useful. PLoS Medicine 13: e1002049.

Janssen J, Hale L, Mirfin-Veitch B, et al. (2013) Building the Research Capacity of Clinical Physical Therapists Using a Participatory Action Research Approach. Physical Therapy 93: 923-934.

Kitson A. (2010) The knowledge-to-action cycle: identifying the gaps. CMAJ: Can Med Assoc J 182.

Kitson A, Brook A, Harvey G, et al. (2017) Using complexity and network concepts to inform healthcare knowledge translation. Int J Health Policy Manag. 6: 1-13.

Kitson A, Powell K, Hoon E, et al. (2013) Knowledge translation within a population health study: how do you do it? Implement Sci 8.

LaRocca R, Yost J, Dobbins M, et al. (2012) The effectiveness of knowledge translation strategies used in public health: a systematic review. BMC Public Health 12: 751.

Lewis $S$ and Russell A. (2011) Being embedded: A way forward for ethnographic research. Ethnography 12: 398-416.

Marshall M. (2014a) Bridging the ivory towers and the swampy lowlands; increasing the impact of health services research on quality improvement. International Journal for Quality in Health Care 26: 1-5.

Marshall M. (2014b) Researchers-in-Residence: a solution to the challenge of evidence-informed improvement? Primary Health Care Research \& Development 15: 337-338.

Marshall M, Eyre L, Lalani M, et al. (2016) Increasing the impact of health services research on service improvement: the researcher-in-residence model. J $R$ Soc Med 109: 220-225.

Marshall M, Pagel C, French C, et al. (2014) Moving improvement research closer to practice: the Researcher-in-Residence model. BMJ Quality \&amp; Safety 23: 801-805.

Matus J, Walker A and Mickan S. (2018) Research capacity building frameworks for allied health professionals - a systematic review. BMC Health Services Research 18: 716.

McGinity R and Salokangas M. (2014) Introduction: 'embedded research' as an approach into academia for emerging researchers. Management in Education 28: 3-5.

Mickan S and Coates D. (under review) Aim and purpose of the embedded researcher role: Current perspectives

International journal of health planning and management.

Miller S, Abalos E, Chamillard M, et al. (2016) Beyond too little, too late and too much, too soon: a pathway towards evidence-based, respectful maternity care worldwide. The Lancet 388: 2176-2192.

Nilsen P. (2015) Making sense of implementation theories, models and frameworks. Implementation Science 10: 53.

Olswang LB and Prelock PA. (2015) Bridging the Gap Between Research and Practice: Implementation Science. Journal of Speech, Language \& Hearing Research 58: S1818-S1826.

Pellecchia M, Mandell DS, Nuske HJ, et al. (2018) Community-academic partnerships in implementation research. Journal of community psychology 46: 941-952.

Perry L, Grange A, Heyman B, et al. (2008) Stakeholders' perceptions of a research capacity development project for nurses, midwives and allied health professionals. Joournal of nursing management 16: 315-326.

Powell K, Kitson A, Hoon E, et al. (2013) A study protocol for applying the co-creating knowledge translation framework to a population health study. Implementation Science 8: 98.

REDCap. (2020) Available at: https://www.project-redcap.org/ (accessed 18/05/2020).

Runciman W, Hunt T, Hannaford N, et al. (2012) CareTrack: assessing the appropriateness of health care delivery in Australia. . Medical Journal of Australia 197.

Rycroft-Malone J, Wilkinson J, Burton C, et al. (2013) Collaborative action around implementation in Collaborations for Leadership in Applied Health Research and Care: Towards a programme theory. Health Services Research \& Policy 18: 13-26.

Schaffer MA, Sandau KE and Diedrick L. (2013) Evidence-based practice models for organizational change: overview and practical applications. J Adv Nurs 69: 1197-1209. 
Sim M, Kirk S, Aiken A, et al. (2018) The embedded research experience and population health system impact in Nova Scotia. Healthy Living, Healthy Life: Collaborative Health Conference on Research, Practice and Community Innovations Conference. Dalhousie University, Halifax.

Stetler CB, Damschroder L, Helfrich CD, et al. (2011) A guide for applying a revised version of the PARIHS framework for implementation. Implement Sci 6.

Tran N, Langlois E, Reveiz L, et al. (2017) Embedding research to improve program implementation in Latin America and the Caribbean. Rev Panam Salud Publica e75:41.

Vindrola-Padros C, Eyre L, Baxter H, et al. (2019) Addressing the challenges of knowledge coproduction in quality improvement: learning from the implementation of the researcher-inresidence model. 28: 67-73.

Vindrola-Padros C, Pape T, Utley M, et al. (2017) The role of embedded research in quality improvement: a narrative review. BMJ Quality \& Safety 26: 70-80.

Walsh A. (2011) Beyond a naturally occurring ethnography: the work-based researcher. Higher Education, Skills and Work-Based Learning 1.

Ward V, House A and Hamer S. (2009) Knowledge Brokering: The missing link in the evidence to action chain? Evidence \& policy : a journal of research, debate and practice 5: 267-279.

Wenke R and Mickan S. (2016) The role and impact of research positions within health care settings in allied health: a systematic review. BMC Health Services Research 16: 355.

Wenke R, Ward EC, Hickman I, et al. (2017) Allied health research positions: a qualitative evaluation of their impact. Health Research Policy and Systems 15.

Williams NJ, Wolk CB, Becker-Haimes EM, et al. (2020) Testing a theory of strategic implementation leadership, implementation climate, and clinicians' use of evidence-based practice: a 5-year panel analysis. Implementation Science 15: 10. 